\title{
Natural vibration characteristics analysis of single-layer valley type latticed intersected cylindrical shell
}

\author{
Xiaoyang $\mathrm{Lu}^{1,}$, , Xiong Jiang ${ }^{2, b}$,Shaobo $\mathrm{Lu}^{3, \mathrm{c}}$, Haoxin $\mathrm{Fu}^{2, \mathrm{~b}}, \mathrm{Tao}_{\mathrm{Li}} \mathrm{L}^{1,}$, \\ Lulu Wang ${ }^{1, a}$ \\ ${ }^{1}$ Research Institute of Engineering Mechanics, Shandong Jianzhu University, Jinan 250101,China \\ ${ }^{2}$ Civil Engineering School, Shandong Jianzhu University,Jinan 250101,China \\ ${ }^{3}$ Shandong Tongyuan Design Group Co. Ltd. ,Jinan 250101,China \\ aluxy5504@163.com., b821985898@qq.com, c946761893@qq.com
}

\begin{abstract}
Keywords:single-layer valley type latticed intersected cylindrical shell ; natural vibration characteristics; natural vibration frequency

Abstract. Using ANSYS and subspace iteration method to analyze natural vibration frequencies of Three-directional grid LICS,Fred Boolean type LICS,Single-diagonal LICS,Double-diagonal LICS .The figures of natural frequencies and corresponding shapes are obtained.Macro parameters include span $S$, rise $f$, valley number $K n$, radial node number $N x$ on natural vibration frequencies are analyzed under 52 working conditions. The results show that natural vibration characteristics of There-grid LICS is the best and Fred Boolean type LICS,Single-diagonal LICS and Double-diagonal LICS' are close.It provides reference for practical engineering design.
\end{abstract}

\section{Introduction}

The strong ground motion makes reticulated shells vibrate when earthquake happens. For vibrated reticulated shells, the effect of inertia is not negligible in general.The inertia effect caused by seismic makes reticulated shells produce a great deal of seismic internal force and displacement, which is likely to cause the destruction or collapse for reticulated shells[1].Therefore, it is very important to analyze the seismic response of reticulated shells in seismic precautionary zone[2]. The frequency of natural vibration which is the inherent property of reticulated shells directly influences dynamic characteristics under earthquake.It is necessary to study characteristics of reticulated shells' natural vibration in seismic response analysis. This paper studies modal analysis of Three-directional grid LICS,Fred Boolean type LICS,Single-diagonal LICS,Double-diagonal LICS by using ANSYS.The influence of $\operatorname{span}(S)$, rise $(f)$, valley number $(K n)$ and radial node number $(N x)$ on LICS's frequency is studied on the base of natural frequencies and shapes are obtained.

\section{Basic structural information}

The Q235 hot rolled seamless bars are used in the structure. The modulus of elasticity is $2.06 \times 10^{5}$ MPa.The Poisson ratio is 0.3. The element of beam188[3] of ANSYS is used in the modeling. The hinged supports are used in the structure which has $235 \mathrm{MPa}$ of yield strength. The average roof's loads are $2.35 \mathrm{kN} / \mathrm{m}^{2}$ [4] which divided by g to be applied on the corresponding joints. The bars' cross section of different parts of the single LICS are different[[5,6]. The hot rolled seamless bars with 351 $\times 16 \mathrm{~mm}$ are selected on the valley line and the outermost hoop and the others is $299 \times 16 \mathrm{~mm}$.

\section{Structural natural vibration characteristics}

Using subspace iteration method[7] to analyze natural frequencies and corresponding patterns which are obtained by modal analysis of ANSYS[8] of Three-directional grid LICS,Fred Boolean type LICS,Single-diagonal LICS,Double-diagonal LICS.Characteristics of natural vibration of Three-directional grid LICS,Fred Boolean type LICS,Single-diagonal LICS and Double-diagonal LICS are analyzed in the paper. 
Forty orders of frequencies are get in ANSYS[9] when LICS have specifical spans $(55 \mathrm{~m})$,rises $(10 \mathrm{~m})$, different valley numbers(6) and node numbers(6). The roof's even loads which are $2.35 \mathrm{kN} / \mathrm{m}^{2}$ divided by $\mathrm{g}$ are applied on the corresponding joints[10]. The model uses mass 21 of ANSYS.Forty orders of frequencies of natural vibration of Three-directional grid LICS,Fred Boolean type LICS,Single-diagonal LICS,Double-diagonal LICS in table 1.Three-directional grid LICS's graph of eight orders of mode shape are in Fig.1.

Table1 Forty orders of frequencies of natural vibration of LICS $(\mathrm{Hz})$

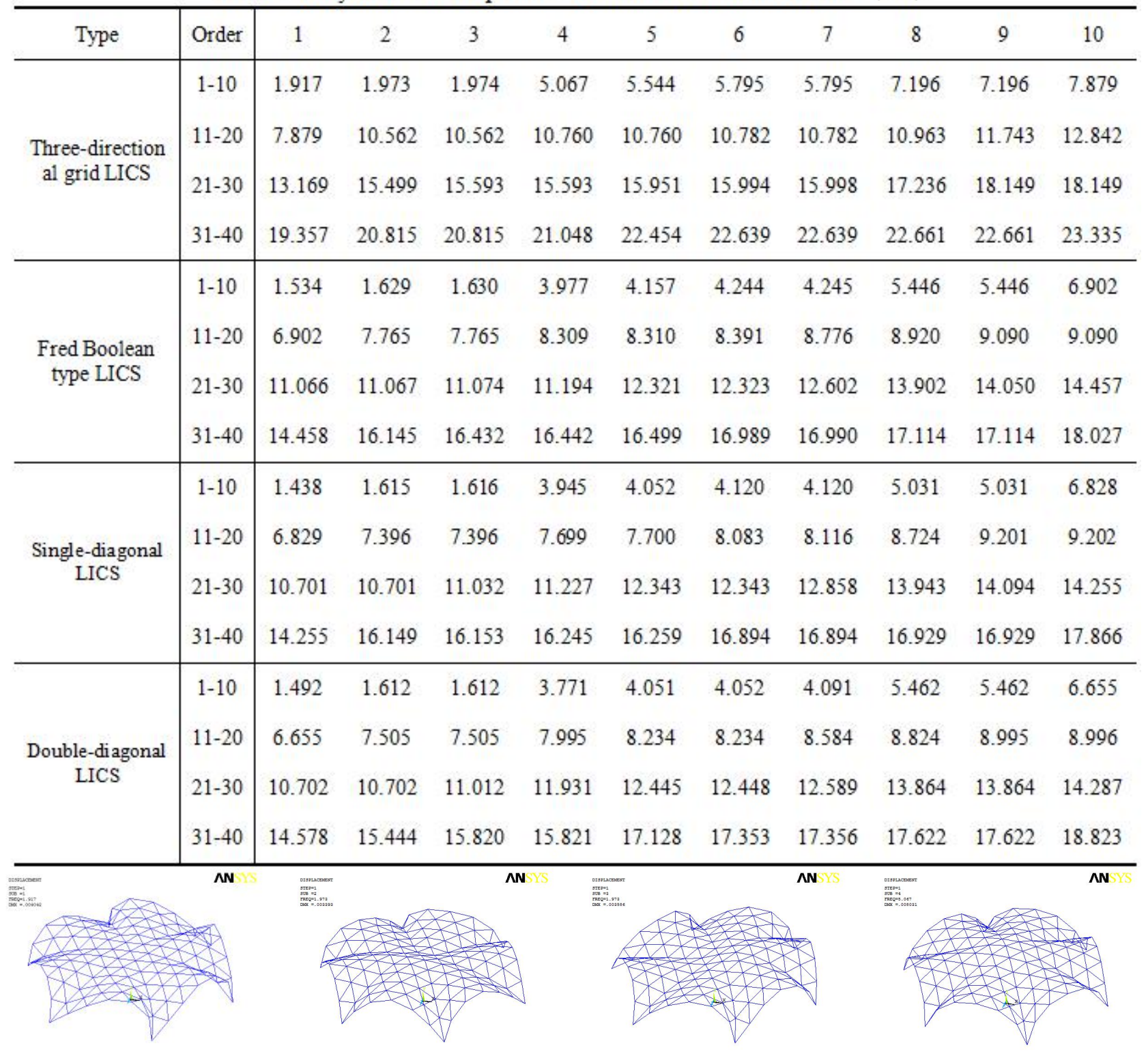

The first order

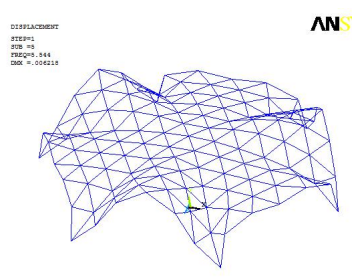

The fifth order
The second order

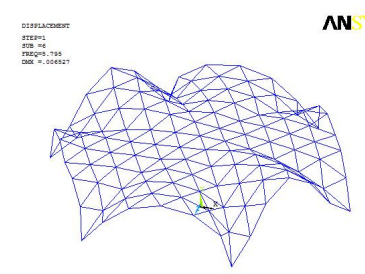

The sixth order
The third order

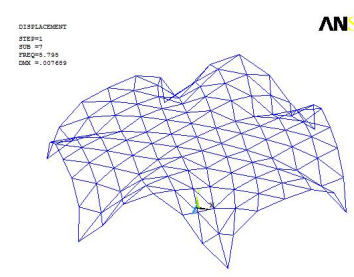

The seventh order

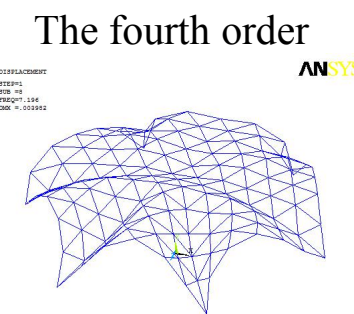

The eighth order

Fig.1 Three-directional grid LICS's graph of eight orders of mode shape

The following conclusions can be drawn from table 1 and Fig. 1 under working conditions.

The vibration patterns of Three-directional grid LICS,Fred Boolean type LICS,Single-diagonal LICS and Double-diagonal LICS are characterized by the horizontal vibration patterns, the vertical 
vibration patterns, combinations of horizontal and vertical vibration patterns. So it is necessary to input the horizontal and vertical seismic wave in earthquake response analysis.

Three-directional grid LICS's frequencies of natural vibration are greater than Fred Boolean type LICS,Single-diagonal LICS and Double-diagonal LICS' in the same order,so is the rigidity.The frequencies of natural vibration of Fred Boolean type LICS,Single-diagonal LICS and Double-diagonal LICS' are close because their bars' connections are roughly the same. Three-directional grid LICS,Fred Boolean type LICS,Single-diagonal LICS and Double-diagonal LICS' frequencies of natural vibration are discrete and some of them are equal because single-layer valley type LICS have multiple symmetry axes.

The first order and the third order patterns of vibration of Three-directional grid LICS are horizontal vibration in the direction of approximately 45 and 60 degrees with the XZ plane.However,the second and the fourth are the vertical vibration along the $\mathrm{Z}$ axis. It is shown that the combinations of horizontal and vertical vibration patterns from the beginning of the fifth order.The surfaces of vibration of the first four orders are simple,the following orders are complicated.

\section{Influence of span on frequency of natural vibration}

The influence of span on frequency of natural vibration of four different kinds of single-layer valley style LICS which have specifical spans $(50,55$ and $60 \mathrm{~m})$, rises $(10 \mathrm{~m})$,different valley numbers $(6)$ and node numbers(6) are analyzed.The roof's even loads which are $2.35 \mathrm{kN} / \mathrm{m}^{2}$ divided by g are applied on the corresponding joints. The model uses mass 21 of ANSYS. The results are drawn in Fig.2.

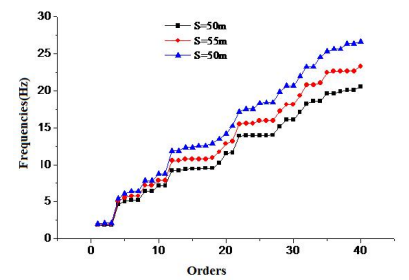

(a)Three-directional grid LICS

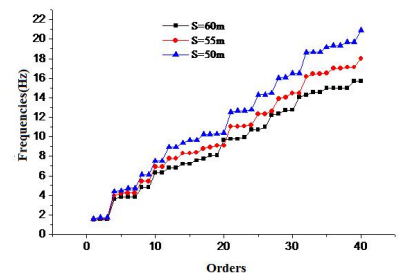

(b)Fred Boolean type LICS

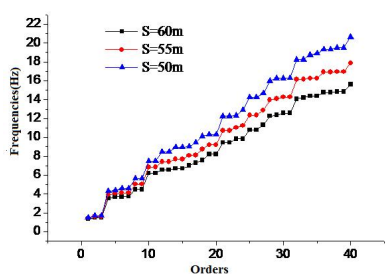

(c)Single-diagonal LICS

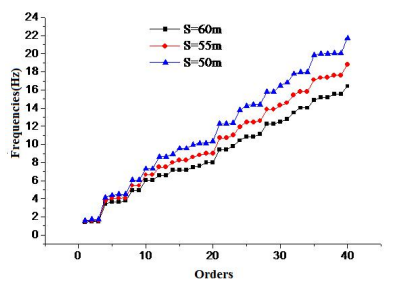

(d)Double-diagonal LICS

Fig.2 The curve of influence of span on frequency

Under 12 working conditions,we know that Three-directional grid LICS,Fred Boolean type LICS,Single-diagonal LICS and Double-diagonal LICS' frequencies of natural vibration which are discrete decrease with span increased, so is the rigidity.At the moment,more energies are absorbed by the structure and the ability to resist deformation is weaker when the earthquake happens.

\section{Influence of rise on frequency of natural vibration}

The influence of rise on frequency of natural vibration of four different kinds of single-layer valley style LICS which have specifical $\operatorname{spans}(55 \mathrm{~m})$,rises $(6,8$ and $10 \mathrm{~m})$,different valley numbers $(6)$ and node numbers(6) are analyzed.The roof's even loads which are $2.35 \mathrm{kN} / \mathrm{m}^{2}$ divided by g are applied on the corresponding joints. The model uses mass 21 of ANSYS. The results are drawn in Fig.3.

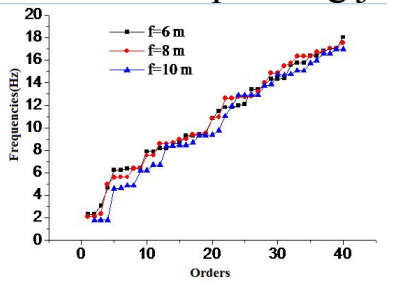

(a)Three-directional grid LICS

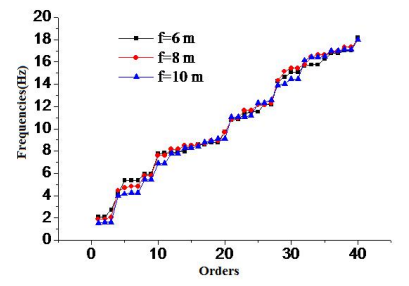

(b)Fred Boolean type LICS

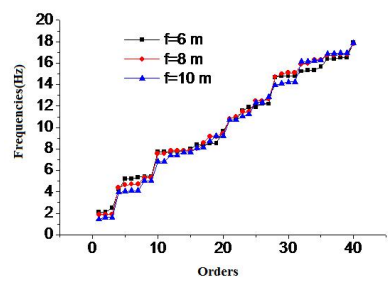

(c)Single-diagonal LICS

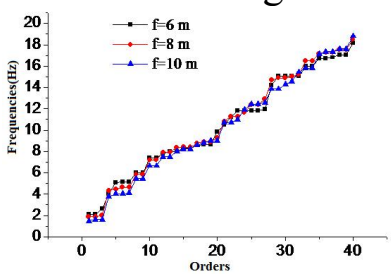

(d)Double-diagonal LICS

Fig.3 The curve of influence of rise on frequency 
Under 12 working conditions,we know that Three-directional grid LICS,Fred Boolean type LICS,Single-diagonal LICS and Double-diagonal LICS' the first ten orders' frequencies of natural vibration decrease with rise increased, so is the rigidity.At the moment,more energies are absorbed by the structure and the ability to resist deformation is weaker when the earthquake happens.Eleven to thirty orders' frequencies of natural vibration are complicated with rise increased.

\section{Influence of $K n$ on frequency of natural vibration}

The influence of $K n$ on frequency of natural vibration of four different kinds of single-layer valley style LICS which have specifical spans $(55 \mathrm{~m})$,rises $(10 \mathrm{~m})$,different valley numbers $(4,5$ and 6$)$ and node numbers(6) are analyzed.The roof's even loads which are $2.35 \mathrm{kN} / \mathrm{m}^{2}$ divided by g are applied on the corresponding joints. The model uses mass 21 of ANSYS. The results are drawn in Fig.4.

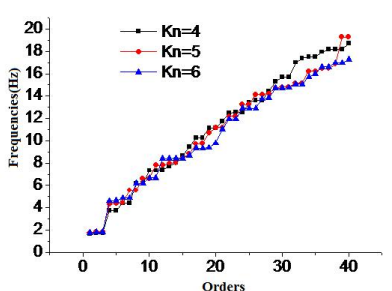

(a)Three-directional
grid LICS

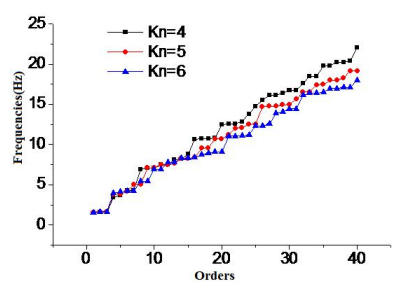

(b)Fred Boolean type LICS

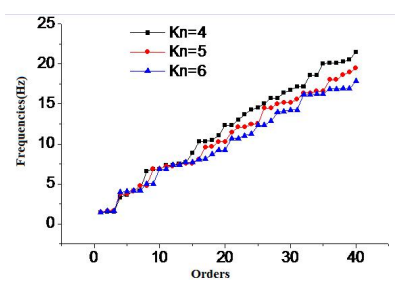

(c)Single-diagonal LICS

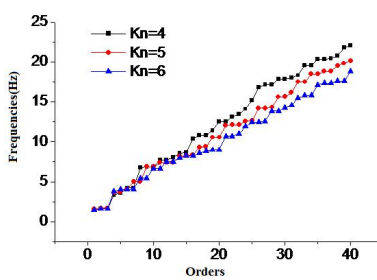

(d)Double-diagonal LICS

Fig.4 The curve of influence of $K n$ on frequency

Under 12 working conditions,we know that Three-directional grid LICS,Fred Boolean type LICS,Single-diagonal LICS and Double-diagonal LICS' frequencies of natural vibration which are discrete decrease with $K_{n}$ increased, so is the rigidity. At the moment,more energies are absorbed by the structure and the ability to resist deformation is weaker when the earthquake happens.

\section{Influence of $N x$ on frequency of natural vibration}

The influence of $N x$ on frequency of natural vibration of four different kinds of single-layer valley style LICS which have specifical spans $(40 \mathrm{~m})$,rises $(10 \mathrm{~m})$, different valley numbers $(6)$ and node numbers $\left(6,11\right.$ and 16) are analyzed.The roof's even loads which are $2.35 \mathrm{kN} / \mathrm{m}^{2}$ divided by gare applied on the corresponding joints. The model uses mass 21 of ANSYS. The results are drawn in Fig.5.

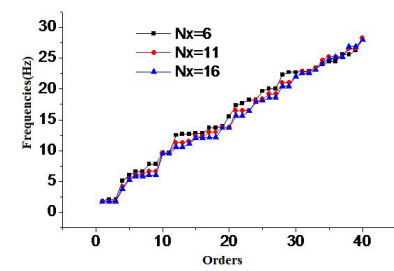

(a)Three-directional grid LICS

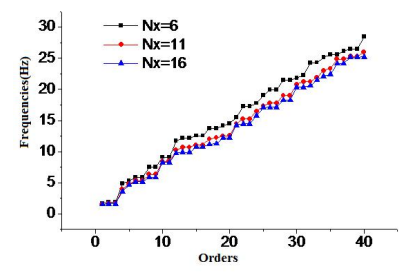

(b)Fred Boolean type LICS

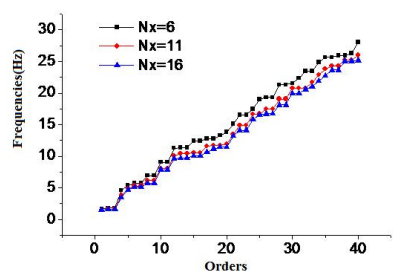

(c)Single-diagonal LICS

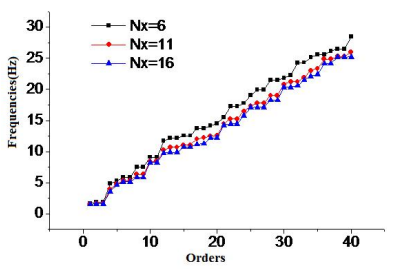

(d)Double-diagonal LICS

Fig.5 The curve of influence of $N x$ on frequency

Under 12 working conditions,we know that Three-directional grid LICS,Fred Boolean type LICS,Single-diagonal LICS and Double-diagonal LICS' frequencies of natural vibration which are discrete decrease with $N x$ increased, so is the rigidity. At the moment,more energies are absorbed by the structure and the ability to resist deformation is weaker when the earthquake happens. 


\section{Conclusions}

This paper analyzes four influence factors and 52 working conditions on natural vibration frequency of four different kinds of single-layer valley style latticed intersected cylindrical shell.The results are as follows.

The vibration patterns of single-layer valley style latticed intersected cylindrical shell are characterized by the horizontal vibration patterns, the vertical vibration patterns, combinations of horizontal and vertical vibration patterns.Three-directional grid LICS's frequencies of natural vibration are greater than Fred Boolean type LICS,Single-diagonal LICS and Double-diagonal LICS' in the same order. The frequencies are discrete and some of them are equal.

The natural vibration frequencies of Three-directional grid LICS,Fred Boolean type LICS,Single-diagonal LICS and Double-diagonal LICS decrease with span,rise,valley number and radial node number increased.Rise has little influence on natural vibration frequency.

It is suggested that Three-directional grid LICS should be first considered in practical engineering design.

\section{Acknowledgements}

This work was financially supported by projects of Shandong postgraduate innovation (SDYY08038) and postgraduate high quality courses of Shandong Jianzhu University (YZKC201605).

\section{References}

[1] Chang Wu,Xiuli Wang,Kun Zhou.Dynamic failure analysis of K6 vierendeel reticulated spheric al shell under sever earthquakes. Spatial Structures,2015,(02):60-65.

[2] Zhihong Li,Hongyan Zhang,Jian Liu,Dengfeng Peng .Analysis of natural vibration characteristi cs of bracket set reticulated shell. Steel Structure, 2012,(12):7-10.

[3] Xinmin Wang. Numerical analysis of ANSYS engineering structure. Beijing China Communicat ion Press, 2014.

[4] GB 50009-2012 Load code for the design of building structures.Beijing:China Architecture\&Building Press,2012.

[5] Ya Su,Shao bo Lu,Xiaoyang Lu, et al.Parametric modeling and shape optimization of hybrid saddle reticulated shell.Journal of Shandong Jianzhu University,2016,01:38-46.

[6] Shiying Chen,Xiaoyang Lu,Haiyan Zhu.The effect of bar group and mesh density on shell optimization design for gross section. Mechanics in Engineering,2011,02:71-74.

[7] Zhihua Chen,Hongbo Liu,Ting Zhou,Xiuzhu Qu.Parametric calculation and analysis of spatial steel structure based on APDL.China WaterPower Press,2013.

[8] Zhihua Chen,Jingjing Bai.Roof structural modeling of Dalian polar region marine museum and static and dynamic analysis on zigzag cone lattice shell.Progress in Steel Building Structures,2012, (01):1-7.

[9] Zhihua Chen,Bingbing Chen. Structural design and analysis of an single-layer reticulated shell supported by spatial arc trusses.Building structure,2014,(10):51-55.

[10] Shuguang Gong, Guilan Xie.Parametric programming and command manual of ANSYS.China Machine Press,2010. 